\title{
INDEPENDENT KNOTS IN BIRKHOFF INTERPOLATION ${ }^{1}$
}

\author{
BY G. G. LORENTZ
}

Communicated by Alberto P. Calderón, April 8, 1976

We consider Birkhoff interpolation for an incidence matrix $E=\left(e_{i k}\right)_{i=1}^{m} ; \underset{k=0}{n}$, the "polynomials" $P=\Sigma_{0}^{n} a_{k} u_{k}(x)$, for a system $U=\left\{u_{k}\right\}_{0}^{n}$ of functions $u_{k} \in$ $C^{n}[a, b]$ (or $P=\left\{x^{k}\right\}_{0}^{n}$ ) and the knots $X=\left(x_{1}, \ldots, x_{m}\right)$ satisfying $a \leqslant x_{1}$ $<\cdots<x_{m} \leqslant b$. The method of independent knots appears for the first time in [4]; it is somewhat related to the coalescence method [1], [3].

A function $f \in C^{n}[a, b]$ is annihilated by $E, X$ if

$$
f^{(k)}\left(x_{i}\right)=0 \quad \text { for all }(i, k) \text { with } e_{i k}=1 .
$$

From zeros of $f$ and its derivatives given by (1), one can derive further zeros by means of Rolle's theorem. This leads to the following definition. A Rolle set $R$ for a function $f$ annihilated by $E, X$ is a collection $R_{k}, k=0, \ldots, n$, of Rolle sets of zeros (with multiplicities) of the $f^{(k)}$. The sets $R_{k}$ are defined inductively: $R_{0}$ consists of the zeros of $f$ given by (1); if $R_{0}, \ldots, R_{k}$ have been defined, we select $R_{k+1}$-some of the zeros of $f^{(k+1)}$-as follows: $(\alpha) R_{k+1}$ contains all zeros of $f^{(k)}$ of multiplicity $>1$, their multiplicities reduced by 1 . ( $\left.\beta\right) R_{k+1}$ contains all zeros of $f^{(k+1)}$ (with multiplicities) given by (1). $(\gamma)$ For any two adjacent zeros $\alpha, \beta \in R_{k}$ we select a zero $\gamma$ of $f^{(k+1)}$ by means of Rolle's theorem, provided one exists not listed in (1). This new zero $\gamma$ may be different from the $x_{i}$; it may be one of the $x_{i}$, but not listed in (1) as a zero of $f^{(k+1)}$; finally, $\gamma$ may appear as an additional multiplicity of a zero $x_{i}$ of $f^{(k+1)}$ by (1). In this case, $e_{i, k+1}=\cdots=e_{i, k+t}=1, e_{i, k+t+1}=0$. If no zero $\gamma$ as specified exists, there is a loss. $(\delta)$ We adjust the multiplicities in the last case of $(\gamma)$ : if also $e_{i, k+t+2}=\cdots=e_{i, k+s+1}=0$, then $\gamma$ belongs to $R_{k+1}$ with multiplicity s. A Rolle set constructed without losses is maximal. A function $f$ annihilated by $E, X$ may have several Rolle sets, some of them maximal, others are not. Let $m_{\boldsymbol{k}}$ be the number of ones in the column $k$ of $E$, let

$$
\mu_{k}=\left(\cdots\left(\left(m_{0}-1\right)_{+}+m_{1}-1\right)_{+}+\cdots+m_{k-1}-1\right)_{+}+m_{k} .
$$

LEMMA 1. The number of distinct Rolle zeros of $f^{(k)}$ in a maximal Rolle set is exactly $\mu_{k}$.

Let $E$ be a Birkhoff matrix, let $E^{0}$ be derived from $E$ by replacing a one,

AMS (MOS) subject classifications (1970). Primary 41A05; Secondary 15A36.

${ }^{1}$ Research partially supported by NSF grant MPS 75-09833. 
$e_{i_{0} q}=1,1<i_{0}<m$ by zero, let $E^{\prime}, E^{\prime \prime}$ consist of rows $1, \ldots, i_{0}$ and $i_{0}, \ldots$, $m$ of $E^{0}$. Let $\mu_{k}^{0}, \mu_{k}^{\prime}, \mu_{k}^{\prime \prime}$ be defined for the matrices by (2).

LEMMA 2. If $e_{i_{0} q}=1$, one has $\tau=\mu_{q}^{0}-\mu_{q}^{\prime}-\mu_{q}^{\prime \prime}>0$, and if, in addition, $e_{i_{0}-1, q}=0$, then $\tau>0$.

A set $Y \subset[a, b]$ is independent with respect to $U$ if for each $X \subset Y$, each polynomial $P$ annihilated by $E, X$ has a maximal Rolle set. Results on independent sets are based on inequalities of Markov type and on

Lemma 3. For each $l>0$ there is a number $d>0$ with the property that if $P(\alpha)=P(\beta)=0, \beta-\alpha>l$, then at least one point $\alpha+d<\xi<\beta-d$ satisfies $P^{\prime}(\xi)=0$.

THEOREM 1. There exist independent sets $Y=\left\{y_{s}\right\}_{s=-\infty}^{+\infty}$ so that $a<$ $\cdots<y_{-s}<\cdots<y_{s}<\cdots<b$; the $y_{s}$ can be defined inductively; at each step it is enough to take $y_{s}\left(\right.$ or $y_{-s}$ ) sufficiently close to $b$ (or to a).

THEOREM 2. If $Y=\left\{y_{s}\right\}_{s=-\infty}^{+\infty}$ is an independent set, there exist points $z_{s t}$ in $\left(y_{s}, y_{s+1}\right)$ so that the set formed by all $z_{s t}$ and all $y_{s}$ is independent.

Lemma 3. Let $1 \leqslant s<i_{0}<t \leqslant m$. There exists an independent set $\left(x_{1}, \ldots, x_{m}\right)$ and an interval $I=[c, d] \subset\left(x_{i_{0}-1}, x_{i_{0}+1}\right)$ so that: (i) If $P$ is annihilated by $E, X$, then Rolle zeros of $P$ are derived only from $x_{i}, s \leqslant i \leqslant t$; (ii) problem (1) for $E, X$ is regular if $x_{i_{0}} \in I$, and row $i_{0}$ of $E$ is consevrative.

By means of these results we can estimate the number of changes of signs of determinants $D_{E}(X)$ of (1). Let $U=P$.

THEOREM 3. If $X$ is as in Lemma 3, and if row $i_{0}$ of $E$ has exactly one odd supported sequence beginning with $e_{i_{0}, q}=1$, then, as $x_{i_{0}}$ moves from $c$ to $d, D_{E}(X)$ changes sign at least $\tau$ times. If $X^{\prime}, X^{\prime \prime}$ have $x_{i_{0}}$ in the extreme positions,

$$
\operatorname{sign} D_{E}\left(X^{\prime}\right)=(-1)^{\tau} \operatorname{sign} D_{E}\left(X^{\prime \prime}\right)
$$

Corollaries. 1. If $E$ is a Birkhoff matrix, $s=1, t=m$, then $\tau>0$ and $E$ is strongly singular. This is the main theorem of [2], but with a precise number of changes of sign.

2. Assume that row $i_{0}$ consists of disjoint portions $S_{j}, j=1, \ldots, p$, which follow each other. Let matrix $E_{j}$ have rows $s, \ldots, t$ of $E$, with row $i_{0}$ replaced by three rows $S_{1} \cup \cdots \cup S_{j-1}, S_{j}$, and $S_{j+1} \cup \cdots \cup S_{p}$. Let $S_{j}$ have exactly one odd supported sequence in $E_{j}$ with $\tau_{j}$ constructed as in Lemma 2. Then $E$ is strongly singular if $\Sigma \tau_{j}+\sigma$ is odd, where $\sigma$ is the difference of the interchanges of rows for the two coalescences $\left(\cdots\left(S_{1} \cup S_{2}\right) \cup \cdots \cup S_{p}\right) \cup$ $E_{i_{0}+1}$ and $S_{1} \cup\left(S_{2} \cup \cdots \cup\left(S_{p} \cup E_{i_{0}+1}\right) \cdots\right)$. If $s=1, t=m$, this is the criterion [1, Theorem 2.3]. 
3. If $s \neq 1, t \neq m$, we obtain new criteria. One notices the phenomenon that a submatrix $F$ of $E$ may be "so bad" that any of its extensions to a Birkoff matrix is strongly singular.

\section{REFERENCES}

1. S. Karlin and J. M. Karon, Poised and non-poised Hermite-Birkoff interpolation, Indiana Univ. Math. J. 21 (1971/72), 1131-1170. MR 47 \#3877.

2. G. G. Lorentz, Birkoff interpolation and the problem of free matrices, J. Approximation Theory 6 (1972), 283-290. MR 49 \#5639.

3. - Coalescence of matrices, J. Approximation Theory (to appear).

4. G. G. Lorentz and K. L. Zeller, Birkoff interpolation, SIAM J. Numer. Anal. 8 (1971), 43-48. MR $45 \# 4595$.

DEPARTMENT OF MATHEMATICS, UNIVERSITY OF TEXAS, AUSTIN, TEXAS 78712 